\title{
Corela
}

Cognition, représentation, langage

2-2 | 2004

Vol. $2, n^{\circ} 2$

\section{Saillance physique et saillance cognitive}

Frédéric Landragin

\section{OpenEdition}

\section{Journals}

Édition électronique

URL : http://journals.openedition.org/corela/603

DOI : $10.4000 /$ corela.603

ISSN : 1638-573X

\section{Éditeur}

Cercle linguistique du Centre et de l'Ouest - CerLICO

\section{Référence électronique}

Frédéric Landragin, «Saillance physique et saillance cognitive », Corela [En ligne], 2-2 | 2004, mis en ligne le 15 décembre 2004, consulté le 19 avril 2019. URL : http://journals.openedition.org/corela/603 ; DOI : 10.4000/corela.603

Ce document a été généré automatiquement le 19 avril 2019

\section{(c) (i) (9)(2)}

Corela - cognition, représentation, langage est mis à disposition selon les termes de la licence Creative Commons Attribution - Pas d'Utilisation Commerciale - Partage dans les Mêmes Conditions 4.0 International. 


\title{
Saillance physique et saillance cognitive
}

\author{
Frédéric Landragin
}

\section{Introduction}

1 La notion de saillance (ou prégnance) est avant tout liée à l'émergence d'une figure sur un fond. Issue de l'analyse de la perception visuelle, cette notion permet d'expliquer pourquoi nous distinguons des formes là où on ne pourrait voir que du bruit. Appliquée à la perception du langage, cette notion offre de nombreuses perspectives: pourquoi certains éléments linguistiques sont compris et retenus plus facilement que d'autres? Pourquoi certains référents du discours deviennent prépondérants et susceptibles d'être rappelés par la simple mention d'un pronom? Nous nous proposons dans cet article d'explorer ces questions en procédant à un inventaire des facteurs de saillance, avec un parallèle constant entre les aspects linguistiques et les aspects perceptifs. Cet inventaire nous permettra de préciser les notions de saillance physique et de saillance cognitive, ainsi que de proposer quelques jalons pour une modélisation et une meilleure définition de la notion de saillance.

2 Plus précisément, la saillance intervient fortement lors de la lecture d'un texte ou de l'interprétation d'un énoncé en situation de dialogue : mettant en avant un élément du message, elle dirige l'attention du sujet sur cet élément et privilégie sa prise en compte dans le processus d'interprétation, que ce soit au niveau de la détermination du sens ou plus localement lors de la résolution des références et des coréférences. La saillance permet ainsi d'attribuer un référent à un pronom et constitue d'une manière générale une aide à la résolution des anaphores. En production (ou génération), elle peut diriger la construction d'un message autour d'un élément déjà saillant, ou peut au contraire fournir un ensemble de moyens pour rendre saillant un autre élément et le porter de ce fait à l'attention du lecteur ou de l'interlocuteur. La saillance apparaît ainsi comme un facteur prépondérant dès qu'on s'intéresse à la modélisation du sens, aussi bien en interprétation qu'en génération, et jusque dans la réalisation de systèmes de traitement automatique du langage naturel. 
3 La perception visuelle fait largement intervenir la notion de saillance : notre attention s'arrête prioritairement sur les éléments saillants qui ressortent de notre environnement visuel, jusqu'à axer les processus cognitifs sur ces seuls éléments. La saillance naît de causes variées, comme le montre l'exemple suivant : lorsque nous jetons un œil sur une facture suite à des courses dans un supermarché, il arrive qu'un prix élevé attire directement l'attention, sans même nécessiter le parcours détaillé de la liste des articles achetés. La raison peut en être purement visuelle, comme par exemple la présence d'un plus grand nombre de chiffres dans la représentation écrite du prix. Mais à cette cause visuelle s'ajoute probablement une cause cognitive, liée par exemple à la saillance particulière que prend un prix élevé pour un esprit économe.

D'une manière générale, la saillance visuelle ou linguistique constitue un point de départ pour tout message linguistique. Elle reste néanmoins une notion très floue. La saillance visuelle est liée à la nature et à la disposition d'objets dans une scène, sans être clairement définie par rapport à des propriétés visuelles particulières. La saillance linguistique est liée à la façon dont on présente ou traite un message, et recouvre divers aspects de la structure informationnelle (ou structure communicative), sans être clairement définie par rapport aux composants de cette structure que sont les topique, thème et focus. Elle concerne à la fois la prosodie, le lexique, la syntaxe, la sémantique et la pragmatique. De plus, elle ne s'attache pas seulement au langage mais aussi à la tâche, au sujet et à son individualité. Dans le but de clarifier cette notion, nous voulons faire ici un récapitulatif des rapports entre saillance et chacun de ces aspects. Pour cela, nous proposons une distinction entre d'un côté la saillance physique, qui est liée à la trace physique du message visuel ou linguistique, et de l'autre côté la saillance cognitive, qui est liée aux processus cognitifs et aux spécificités du sujet.

Du point de vue physique, être saillant, c'est ressortir en premier de la perception d'un message visuel ou linguistique compte tenu de la forme que prend ce message, c'est-àdire des propriétés de ses composants (et compte tenu des caractéristiques physiologiques de l'organe de perception). Du point de vue cognitif, être saillant, c'est ressortir en premier de la perception d'une scène compte tenu des représentations mentales de l'utilisateur, de ses intentions de perception, de son attention visuelle et de sa mémoire à court terme, de ses expériences personnelles et de ses émotions.

Notre principale proposition est ainsi une classification des facteurs de saillance physique (que l'on peut appeler P-saillance comme saillance physique ou encore saillance perceptive) et des facteurs de saillance cognitive (que l'on peut appeler C-saillance). Après une section décrivant notre méthodologie et positionnant la saillance par rapport aux notions que sont l'attention, la pertinence ou encore le prototype, nous commençons notre classification par les facteurs physiques de saillance linguistique. Ces facteurs sont précisés en deux sous-catégories, les facteurs physiques liés à la forme de l'énoncé (ou de la phrase écrite) et ceux liés à son sens. Chacun de ces points de vue complémentaires peut donner lieu à une définition de la saillance. Du point de vue de la forme, être saillant, c'est ressortir en premier lors de l'audition d'un énoncé (ou de la lecture d'une phrase) compte tenu de la forme que prend cet énoncé, c'est-à-dire des mots qui le composent, de l'ordre et des fonctions grammaticales de ces mots. La saillance dénote alors le degré d'influence de l'attention du sujet par les entités perçues. Du point de vue du sens de l'énoncé, être saillant, c'est ressortir en premier lors de l'audition d'un énoncé, compte tenu du sens immédiatement perceptible de cet énoncé, c'est-à-dire avant tout processus complexe de raisonnement. Toujours pour la saillance linguistique, la classification que 
nous proposons enchaîne sur les facteurs cognitifs. La saillance dénote alors le degré d'influence de l'attention du sujet par les entités perçues et déjà activées dans ses représentations mentales au moment de l'énonciation, ou encore le degré d'influence de l'attention par les entités perçues et dotées d'une signification propre au sujet. Nous réalisons alors le même travail pour la saillance visuelle, avec une classification des facteurs physiques puis des facteurs cognitifs. Dès lors que nous disposons de descriptions des facteurs de saillance linguistique et des facteurs de saillance visuelle, il est intéressant de comparer ces descriptions. La section 8 montre les similarités entre les aspects linguistiques et les aspects visuels, que ce soit pour la saillance physique ou pour la saillance cognitive. Nous pouvons alors discuter du bien-fondé de cette distinction entre P-saillance et $\mathrm{C}$-saillance, ainsi que du caractère global de la notion de saillance, au-delà des modalités linguistique et visuelle.

\section{Méthodologie et positionnement}

7 Quelle est l'unité à laquelle s'applique la saillance? Pour ce qui concerne la saillance linguistique, le point de départ est l'énoncé perçu ou la phrase lue. Sont ainsi favorisés les aspects formels, tels que les facteurs de saillance liés aux mots et à l'ordre de ceux-ci dans l'énoncé. De par sa nature, un nom propre peut par exemple être considéré comme plus saillant qu'une préposition ou un adverbe, de même que le mot ou groupe de mots amorçant l'énoncé peut être considéré comme plus saillant qu'un mot placé au milieu. Les mots sont aussi des indices conduisant au sens, ce sens concourant à la saillance. Les saillances relatives du sujet et du complément d'un verbe peuvent ainsi dépendre de la sémantique de ce verbe. Enfin, l'utilisation de certains mots compte tenu du contexte révèle certains indices cognitifs. En tenant compte de ces aspects, on peut se demander quelle est l'unité à laquelle s'applique la saillance: est-ce le mot, le groupe de mots, l'entité du discours derrière le mot, ou le sens du mot, c'est-à-dire une représentation construite à partir du mot et de ses rapports avec les autres éléments de l'énoncé ?

Une analogie avec la saillance visuelle peut s'avérer utile pour répondre à cette question. Dans une scène visuelle, il arrive qu'un objet se distingue des autres, par exemple parce qu'il est le seul de sa catégorie ou de sa couleur. Un fauteuil en cuir dans un groupe de chaises en bois est ainsi visuellement saillant. La représentation graphique du fauteuil est comparable à un groupe de mot (fauteuil en cuir); le fauteuil lui-même, en tant que référent, est comparable à l'entité de discours; et la scène visuelle dans laquelle ce fauteuil se distingue est comparable à la scène verbale construite par le discours, dans le sens de (Victorri \& Fuchs, 1996) et plus généralement selon les principes des grammaires cognitives. Comme nous l'avons décrit dans (Landragin, 2004) et comme nous le verrons dans les sections 6 et 7, la saillance visuelle s'applique aux objets, sa détermination tenant compte à la fois des propriétés des représentations graphiques, des propriétés attachées aux objets, de la configuration de la scène, ainsi que, dans une certaine mesure, des états mentaux du sujet impliqué dans la situation. Il nous semble de même que la saillance linguistique s'applique aux entités du discours et pas aux seuls mots de l'énoncé qui sont trop réducteurs. Sa détermination fait également appel à tous les aspects cités.

9 Nous en déduisons notre méthodologie d'étude de la saillance linguistique : nous partons des mots de l'énoncé, nous déterminons les entités du discours, et nous leur attachons une saillance qui tient compte de l'ensemble des éléments présents dans l'énoncé et des conclusions que nous pouvons tirer sur sa structure et sur son sens. Dans l'exemple (1), 
nous partons ainsi des expressions référentielles le triangle rouge et le bleu, et nous nous intéressons aux saillances relatives des deux entités du discours correspondantes, en tenant compte du prédicat qui les lie et de la structure informationnelle de l'énoncé. Même si la notion de scène verbale peut s'avérer utile dans ce processus, nous essayerons de nous en passer et de nous focaliser sur les composantes explicites de l'énoncé de départ, en faisant le moins d'hypothèses possible.

(1) Le triangle rouge doit se mettre à côté du bleu

10 En attachant ainsi la saillance aux entités du discours, nous ne donnons une importance que secondaire aux aspects cognitifs. L'intention du sujet est ainsi considérée comme s'appliquant à des entités particulières, et l'étiquette "saillant dans la structure intentionnelle » ne se rattache à ces entités qu'au moment de la détermination de la saillance. Ce choix nous semble être en adéquation avec l'approche consistant à partir de l'énoncé pour élaborer des hypothèses sur la cognition du sujet: les aspects cognitifs ne sont que des hypothèses et ne doivent ainsi pas constituer un point de départ méthodologique.

11 Pour mieux définir la saillance, il nous semble important de clarifier les distinctions que nous faisons entre cette notion et d'autres notions comme celles d'attention, de pertinence et de prototype, qui sont souvent confondues avec elle. La saillance et l'attention interviennent de manière complémentaire dans le processus de perception. Comme le détaille (Rousselet \& Fabre-Thorpe, 2003) dans un contexte visuel facilement transposable au langage, notre perception est dirigée d'un côté par des modulations attentionnelles descendantes (top-down), et de l'autre côté par des modulations ascendantes (bottom-up). La saillance constitue la principale modulation ascendante, et interagit ainsi avec l'attention selon une même dimension mais dans des directions opposées. L'attention du sujet sur une certaine entité peut augmenter la saillance de cette entité, et, inversement, la saillance d'une entité peut entraîner l'attention du sujet sur celle-ci, mais il n'y a en aucun cas équivalence entre saillance et attention.

Selon (Pattabhiraman \& Cercone, 1990), la saillance et la pertinence relèvent d'un même objectif: "salience and relevance are theoretical notions which are influential in accounting for how or why certain objects, concepts, properties or actions are highlighted or preferred in natural language processing » (p. 80). Même objectif, mais natures différentes: Pattabhiraman considère que la saillance est extérieure au sujet, alors que la pertinence est liée à des facteurs tels que ses buts et sa motivation. En considérant que la saillance dépend de facteurs cognitifs, nous n'allons pas dans ce sens. Pour nous, saillance et pertinence sont toutes deux liées au sujet, et nous faisons la distinction sur un autre plan : la saillance nous semble intervenir à un niveau très proche de la perception, c'est-à-dire au niveau des processus cognitifs inférieurs (attention, perception, mémoire), alors que la pertinence relève des processus cognitifs supérieurs (représentation mentale, raisonnement, jugement, décision, résolution de problèmes). En effet, telle que décrite dans (Sperber \& Wilson, 1995), la pertinence n'a de sens qu'en considérant les résultats de l'interprétation en contexte, et en particulier les inférences effectuées lors de ce processus. Dans la suite, nous resterons ainsi au niveau des processus inférieurs.

13 Enfin, propriété saillante et propriété prototypique d'un objet sont souvent assimilées. S'il est vrai que des caractéristiques saillantes peuvent être à l'origine d'un prototype, et que la connaissance d'un prototype renforce la saillance de certains objets, il n'en reste pas moins que la saillance est liée à un objet précis dans un environnement précis, alors 
que le prototype est lié à une représentation mentale durable et culturelle. Cette interaction est décrite par (Rastier, 1991) qui part de l'exemple de la catégorisation des couleurs : «[les sociétés] utilisent les qualités les plus saillantes perceptivement et qui donc se prêtent le mieux à la discrimination pour articuler leurs codes sémiotiques [...]. En retour, leur codification culturelle renforce et stabilise la saillance des stimuli. » (p. 183). Nous considérons donc que l'influence d'un prototype est l'un des facteurs cognitifs de saillance pouvant intervenir lors de la perception immédiate, et nous ne parlerons de saillance que dans une situation donnée, dans un intervalle de temps précis.

\section{Saillance linguistique : facteurs physiques liés à la forme de l'énoncé}

Notre point de départ étant l'énoncé avec ses caractéristiques linguistiques, nous commençons notre classification des facteurs de saillance par les facteurs physiques, c'est-à-dire ceux qui possèdent une trace linguistique concrète.

15 A la suite de (Stevenson, 2002), nous séparons les aspects formels des aspects sémantiques liés au contenu d'un énoncé. Les aspects formels incluent les caractéristiques lexicales, prosodiques et grammaticales de l'énoncé. Dans la majorité des travaux en linguistique computationnelle, par exemple dans (Alshawi, 1987), (Lappin \& Leass, 1994), ou dans la Théorie du Centrage (Grosz et al., 1995), ce sont essentiellement ces caractéristiques qui définissent la saillance. Les auteurs de la Théorie du Centrage soulignent d'ailleurs que d'autres critères comme la notion de thème ne sont pas pertinents. Comme le rappelle (Krahmer \& Theune, 2002), le critère souvent mis en avant est la récence, c'est-à-dire la proximité textuelle ou temporelle de la dernière mention. Les entités les plus saillantes se réduisent ainsi à celles mentionnées le plus récemment. Nous considérons pour notre part qu'à un niveau formel, la saillance est loin de se réduire à la récence. A partir de ces travaux et des facteurs que nous avons identifiés dans une étude antérieure (Landragin, 2004), nous proposons une classification des facteurs physiques de saillance avec six catégories, chacune faisant l'objet d'une sous-section.

\subsection{La saillance intrinsèque au mot}

16 Certains mots sont saillants de par leur nature même. Dans le cadre du dialogue oral et avec des considérations phonétiques, il s'agit par exemple de mots constitués de phonèmes particulièrement saillants. C'est le cas du mot anglais time avec sa diphtongue qui ressort particulièrement bien. Certains phonèmes peuvent également être saillants grâce à leur rareté ou à une particularité dans leur prononciation, par exemple une combinaison de phonèmes peu fréquente. C'est le cas d'un grand nombre d'onomatopées. Plus généralement, les mots caractérisés par un accent lexical particulier, ainsi que les mots qui contiennent beaucoup d'allitérations ou beaucoup d'assonances sont intrinsèquement saillants. A l'écrit, ce facteur correspond à un mot dont l'orthographe inhabituelle, incluant par exemple une succession rare de consonnes, le rend intrinsèquement saillant.

Certains mots sont saillants de par leur nature grammaticale. On peut ainsi considérer que les noms propres sont saillants. Ce facteur a souvent été évoqué, par exemple dans 
(Garrod \& Sanford, 1988) pour des récits narratifs : «A named character is more highly focused than one described by a referring expression ».

Certains mots sont intrinsèquement saillants de par leur manque d'autonomie référentielle et de l'habitude qu'ils entraînent chez l'interlocuteur à faire particulièrement attention aux conditions de leur énonciation. C'est le cas de mots tels que ça, ainsi que des déictiques purs (ou essentiels) en général.

\subsection{La saillance due à une mise en avant explicite lors de l'énonciation}

19 En nous plaçant au niveau de l'intonation, c'est-à-dire des procédés prosodiques par lesquels une information est mise en premier plan, un premier facteur de saillance dans cette catégorie est la présence d'un accent de focalisation. Comme le détaille (Rossi, 1999), cet accent se réalise par une augmentation brusque de la fréquence fondamentale et de l'intensité. L'élément de l'énoncé oral auquel cet accent s'applique en est rendu saillant, de même que l'entité de discours correspondant à cet élément.

20 Un deuxième facteur de saillance est l'intonation montante, particulièrement pour une phrase interrogative. (Lambrecht, 1994, p. 245) donne trois exemples en français d'un même contenu où l'élément saillant varie grâce à l'intonation interrogative (2). L'élément saillant est toujours le dernier mot, et peut donc être, au choix, le lieu, l'action ou l'interlocuteur.

(2) a. Tu vas où ?

b. Où tu vas?

c. Où vas-tu?

21 D'une manière générale, toute prosodie particulière peut rendre saillant un élément d'un énoncé oral. Qu'il s'agisse d'un accent tonique marqué, d'une lenteur inhabituelle et intentionnelle, de la transmission d'une émotion comme la colère ou l'ironie par une prononciation ou une intonation adéquate, toute prosodie inhabituelle met en avant l'élément auquel elle s'applique. Un autre exemple est la rupture dans une continuité de rythme : lorsque l'énoncé se caractérise par un rythme et que la prononciation d'un mot vient casser ce rythme, le mot en devient saillant. Une rupture dans un rythme monotone, et une irrégularité dans un rythme parfaitement périodique constituent ainsi des causes de saillance. Ce facteur de saillance est particulièrement utilisé en poésie, par exemple dans le vers de Baudelaire (3), où, du fait des accents marqués sur si, vent, prend et mer, le rythme est du type 3-3-2-4, ce qui marque une rupture au niveau de prend qui en devient saillant. Nous noterons que certains facteurs prosodiques ont des équivalents à l'écrit : un accent particulièrement fort se rapproche par exemple d'une typographie en gras ou en italique.

(3) La musique souvent me prend comme une mer

Un autre exemple lié au rythme d'élocution est la présence d'une pause avant et éventuellement après la prononciation d'un mot ou d'un groupe de mots, qui en est rendu saillant. Cette saillance due à un détachement a pour équivalent à l'écrit une mise en apposition, par exemple entre deux virgules ou deux tirets longs.

Un autre facteur de saillance liée à l'énonciation est la présence d'un geste coréférent qui vient appuyer un élément de l'énoncé, le geste pouvant être ostensif, expressif, ou même paraverbal comme le sont les gestes de battement qui rythment les paroles (Cosnier \& 
Vaysse, 1997). Dans les trois cas, il peut s'agir de gestes de la main ou, plus généralement, de la direction du regard, d'une mimique faciale, d'une posture particulière dans la situation de communication. Dans une situation de dialogue, il arrive aussi que l'un des interlocuteurs bénéficie d'une saillance due à la succession des tours de parole. Dans un même ordre d'idée, la saillance liée à l'énonciation peut être renforcée par un marqueur linguistique tel que dis-je, j'insiste ou je vous l'affirme.

Enfin, un dernier facteur de saillance est l'erreur de prononciation, un bégaiement par exemple, qui, bien qu'involontaire, porte un élément de l'énoncé à l'attention de l'interlocuteur. L'équivalent à l'écrit de cet exemple un peu marginal est la faute de frappe ou l'erreur d'orthographe, qui peut parfois être tellement saillante qu'elle en perturbe la lecture.

\subsection{La saillance due à une construction syntaxique dédiée}

La construction syntaxique la plus utilisée pour mettre en avant un élément est sans doute la construction clivée en c'est... qui..., c'est-à-dire avec un présentatif. Le triangle rouge est ainsi rendu saillant dans (4). (Lambrecht, 1994) cite d'autres exemples de constructions syntaxiques dédiées à la saillance : (5a) où la saillance est plus explicite que dans (5b) (p. 14); ou encore les constructions en il y a telle que (6) (p. 144). (Lyons, 1970, p. 258) récapitule à la suite de Hockett quelques dérivations de l'énoncé (7a), où l'un des éléments est rendu saillant, qu'il s'agisse de John (7b) ou du fait de s'enfuir (7c).

(4) C'est le triangle rouge qui doit se mettre à côté du bleu

(5) a. J'ai ma voiture qui est en panne

b. Ma voiture est en panne

(6) Y'a le téléphone qui sonne

(7) a. John ran away

b. The one who ran away was John

c. What John did was run away

Une autre construction dédiée sans marqueur linguistique particulier est le détachement en tête de phrase, comme dans (8) et dans d'autres phrases construites sur la reprise d'un groupe du nom ou d'un groupe de l'adjectif par un pronom (9). Ces constructions à topique sont étudiées dans (Lambrecht, 1996) où sont identifiées des constructions à double topique (10) et des constructions à topique non lié (11). A chaque fois, le but est de rendre saillant le premier groupe nominal. Nous employons ici le mot topique dans son sens le plus syntaxique, c'est-à-dire comme le résultat d'une opération de topicalisation consistant à choisir le premier élément de l'énoncé. Nous verrons plus loin une autre acception de ce terme, avec des considérations sémantiques.

(8) A cette idée je vais en opposer une autre

(9) Le triangle rouge, il doit se mettre à côté du bleu

(10) Jean, sa sœur, je la déteste

(11) La mer, tu vois de l'eau

Une entité du discours peut également être rendue saillante à l'aide d'une construction syntaxique renforcée, telle que (12a) plutôt que (12b) (Lambrecht, 1994, p. 356). La saillance du destinataire à travers la préposition à, mot supplémentaire qui a un rôle de renforcement, est plus forte dans le premier cas que dans le second.

(12) a. Donne-le à lui !

b. Donne-le-lui ! 


\subsection{La saillance syntaxique liée à l'ordre et à la fréquence d'apparition des mots}

des mots et leur place dans une phrase fait intervenir la notion de topologie. D'une manière simplifiée, il est possible de considérer que les positions de début et de fin d'énoncé sont prédisposées pour rendre saillant le mot ou le groupe de mots qui y prend place. Chacune de ces positions autorise également les rejets, comme nous l'avons vu avec le cas des constructions à topique. Dans cette catégorie de facteurs de saillance, nous parlerons de placement à un endroit stratégique comme facteur prépondérant, qu'il s'agisse du début (13a), de la fin (13b), avec éventuellement une construction à rejet (13c). L'intérêt de ces trois exemples par rapport à (13d) est bien entendu de rendre saillant le triangle rouge. Se pose alors la question de l'existence d'une hiérarchie entre les positions de début et de fin. Le critère de récence tend à favoriser la position finale, mais des expériences montrent que le début est souvent plus saillant (Kessler et al., 1996). Il est difficile de faire la part des choses dans la mesure où la position initiale a souvent d'autres fonctions qui sont autant de facteurs de saillance, par exemple la fonction grammaticale sujet.

(13) a. Le triangle rouge doit se mettre à côté du bleu

b. C'est à côté du bleu que tu dois mettre le triangle rouge

c. Tu dois le mettre à côté du bleu, le triangle rouge

d. Tu dois mettre le triangle rouge à côté du bleu moins saillante. Considérons que la notion de constituant recouvre les éléments souscatégorisés par le verbe de l'énoncé, c'est-à-dire principalement le sujet grammatical, le complément d'objet direct et le complément d'objet indirect. Classer ces constituants s'avère nécessaire pour identifier l'élément saillant. Le sujet est ainsi souvent considéré comme le constituant grammatical le plus saillant, particulièrement dans certaines constructions telles que (15) (Sidner, 1979, p. 67). Il se trouve généralement au début de l'énoncé, ce qui renforce la saillance liée à cette position. Les constructions passives, qui permettent d'inverser les fonctions grammaticales, se justifient ainsi. Comme le faisait déjà la thèse de Sidner, la Théorie du Centrage (Grosz et al., 1995) propose une hiérarchie 
des fonctions grammaticales : sujet, puis complément d'objet, puis autres catégories. Pour vérifier la primauté du complément d'objet direct sur les autres types de compléments, Sidner (p. 62) s'appuie sur les exemples (16) et (17) qui sont facilement transposables en français. Dans la suite des deux phrases de l'exemple (16), le problème se pose pour l'attribution d'un référent au pronom it, car she a déjà utilisé le sujet grammatical de la première phrase, autrement dit son constituant le plus saillant. A nickel étant le complément d'objet direct, c'est lui qui va être privilégié dans cette résolution de référence, et non her toy bank. La hiérarchie de saillance va effectivement dans le sens de l'interprétation. Dans l'exemple (17), le complément d'objet direct a meeting va également être privilégié par rapport à Ira dans la résolution de l'anaphore associative the time. Ici aussi, la hiérarchie de saillance favorisant le complément d'objet direct aux autres types de compléments se justifie.

(15) A est un X

(16) Mary took a nickel from her toy bank yesterday

She put it on the table near Bob

(17) I want to schedule a meeting with Ira

The time should be 3 p.m.

Une autre fonction grammaticale, moins fréquente mais constituant un facteur quasiment explicite de saillance linguistique, est la fonction vocative. Garçon est ainsi rendu saillant dans l'exemple (18) de (Lambrecht, 1996). L'auteur montre également que les propriétés des groupes nominaux vocatifs se rapprochent de celles des anaphores et des cataphores nulles (19), renforçant ainsi la saillance de leur antécédent.

(18) Garçon! Il y a une mouche dans ma soupe

(19) Mérite des baffes, ce petit con

\subsection{La saillance indirecte par transfert grammatical de saillance}

Une entité du discours en lien grammatical direct avec l'entité identifiée comme étant la plus saillante voit sa saillance augmenter par transfert. Sans aller trop loin dans cette voie qui risque de rendre saillantes toutes les entités de l'énoncé, nous relèverons deux exemples pour lesquels le transfert est flagrant. Il s'agit premièrement des compléments du nom et de l'adjectif, tels que le complément du nom du sujet grammatical: dans l'exemple (20), le sujet grammatical saillant est une expression référentielle (les employés de la société) qui renvoie à des humains, mais qui rend également saillante la société qui les emploie. Il s'agit deuxièmement d'une succession d'adjectifs venant qualifier un substantif et augmentant ainsi sa saillance. Dans l'exemple qu'il avance (21), (Pattabhiraman, 1993, p.14) montre ainsi que les adjectifs baffling et new viennent renforcer la saillance de galaxy.

(20) Les employés de la société travaillent de nuit

(21) A baffling, new galaxy has been discovered at the centre of the Milky Way

\section{Saillance linguistique : facteurs physiques liés au sens de l'énoncé}

En ce qui concerne les aspects sémantiques nous considérons premièrement la sémantique des mots, deuxièmement la sémantique de l'énoncé avec les notions de rôle thématique, de thème et de topique phrastique, et troisièmement la sémantique du discours avec les notions de topique discursif et de propos. En tenant compte des facettes 
du processus interprétatif et pas seulement de celles de la forme linguistique, nous explorons ici les distinctions classiques suivantes : agent-patient ; thème-rhème ; topiquecommentaire ; posé-présupposé ; connu-nouveau. Les définitions de ces notions s'avèrent très variables et, afin de les délimiter clairement, nous sommes amené à poser quelques principes au fur et à mesure de notre analyse.

\subsection{La saillance liée à la sémantique des mots}

Après avoir détaillé la saillance liée à la forme des mots, nous nous intéressons ici à l'influence des traits sémantiques portés par ces mots sur la saillance des entités du discours correspondantes. (Pattabhiraman, 1993, p. 40) montre ainsi que le trait « humain » ou " animé » augmente la saillance de l'entité correspondante. C'est le cas de la fillette par rapport à la foudre dans l'exemple (22). C'est aussi le cas du possesseur dans les phrases qui, comme celle de l'exemple (23), expriment la possession d'un objet (ibid., p. 61). C'est également le point de vue de la classification de (Lyons, 1980, p. 142) entre personnes, animaux et objets inanimés : « en tant qu'êtres humains nous nous intéressons davantage aux personnes qu'aux animaux, aux animaux qu'aux entités inanimées, et ainsi de suite. Il s'ensuit donc que dans un énoncé composé d'une seule phrase, si l'on se réfère à la fois à une personne et à un animal ou une entité inanimée, l'expression référant à la personne sera en principe thématique, à moins que de bonnes raisons ne s'y opposent ».

(22) La foudre a tué une fillette de huit ans : elle courait sur la plage au beau milieu

de l'orage

(23) Ce livre appartient à Jean : il l'a oublié le mois dernier

Lorsque tous les participants sont des humains, il est également possible d'établir entre eux une hiérarchie de saillance, avant même de prendre en compte la nature de l'événement qui les lie. (Wanner \& Bateman, 1990) s'intéressent ainsi aux influences qu'ils exercent les uns sur les autres. Nous pouvons par exemple considérer qu'un professeur exerce une influence sur son élève et en est plus saillant, qu'il soit l'initiateur de l'action comme dans (24a) ou au contraire un participant secondaire comme dans (24b).

(24) a. Le professeur fait peur à son élève

b. L'élève écoute son professeur

\subsection{La saillance liée au rôle thématique}

Les composants de l'énoncé se caractérisent par un rôle thématique : l'entité du discours qui fait l'action est l'agent, l'entité qui la subit le patient, d'autres rôles thématiques moins importants étant l'instrument, le but, ou encore la localisation. Dans les exemples (25a) et (25b), le triangle rouge est inanimé et ne fait pas l'action : il est statique et on dit qu'il s'agit du thème, notion à ne pas confondre avec celle du thème de la distinction entre thème et rhème. Plus précisément, le thème étudié ici est la relation au verbe d'un groupe nominal qui est l'objet inanimé affecté par l'action du verbe (Sidner, 1979, p. 63).

(25) a. Le triangle rouge est à côté du bleu

b. Le triangle rouge a disparu

(26) The vase broke against the wall

It was covered with many photographs

Il existe plusieurs propositions pour classer selon leur saillance les rôles thématiques. C. Sidner s'intéresse aux phrases comportant un thème et propose de considérer ce rôle comme le plus saillant. C'est le cas dans son exemple (26), où le thème de la première 
phrase (the vase) constitue de par sa saillance un candidat idéal pour une reprise pronominale ultérieure. Si un pronom reprend un autre antécédent, ce qui est le cas avec la deuxième phrase où it reprend the wall, la saillance du thème est telle qu'elle nous incite à imaginer un vase recouvert de photographies! (Sidner, 1979, p. 65). En l'absence de thème, Sidner considère qu'aucun rôle thématique n'est véritablement plus saillant qu'un autre. L'agent, en tout cas, vient en dernier. Dans le même sens, (Stevenson et al., 1994) montre sur la base d'expérimentations qu'entre agent et patient, la préférence va généralement pour le patient, du moins dans certains types de phrases.

Car c'est là toute la difficulté du problème : tout dépend de la phrase, et particulièrement de la catégorie sémantique du verbe. Stevenson et al. (ibid.) montrent ainsi que dans les phrases décrivant un événement, les conséquences de l'événement sont plus présentes dans l'esprit de l'interlocuteur que les conditions initiales. Si ces conséquences s'appliquent à l'agent, celui-ci en devient plus saillant que le patient. (Pearson et al., 2001) détaille le cas des verbes de transfert, et montrent que le receveur est plus saillant que le donneur et que l'objet transféré. Marie est ainsi la personne saillante, aussi bien dans (27a) où elle a le rôle de destinataire que dans (27b) où elle a le rôle d'agent. En ce qui concerne les verbes de causalité, (Garvey \& Caramazza, 1974) puis (Van Kleeck et al., 1988) montrent à l'aide de reprises pronominales que la personne saillante est celle qui est liée à la cause, c'est-à-dire l'agent Sue dans (28a) et le patient Peg dans leur exemple (28b).

(27) a. Jean donne un livre à Marie

b. Marie reçoit un livre de Jean

(28) a. Sue étonne Peg à cause du genre de personne qu'elle est

b. Sue admire Peg à cause du genre de personne qu'elle est

Une méthodologie consiste ainsi à se fonder sur la sémantique verbale pour déterminer le rôle thématique saillant. Comme le montre (Cordier et al., 2000), le travail s'avère ardu car les critères sont nombreux : grammaticalisation, nombre et nature des arguments, aspect, catégorie sémantique du verbe, etc. Beaucoup de recherches sont à faire dans la lignée des considérations précédentes.

\subsection{La saillance liée au thème et au topique de l'énoncé}

41 Au niveau de la sémantique de l'énoncé, les notions de thème et de topique (dans le sens de sentence topic ou de clause topic et non de discourse topic) désignent toutes les deux ce dont l'énoncé parle. C'est le point de départ dans le processus de communication. Selon une expression de Halliday reprise dans (Lyons, 1980, p. 138), c'est le porte-manteau auquel le message est accroché ; c'est l'élément que le locuteur choisit pour construire son message. Le thème s'oppose au rhème, et le topique au commentaire. Dans la suite, nous garderons les termes thème et rhème pour ce qui concerne le niveau de l'énoncé, et nous réserverons le terme propos pour ce qui concerne le topique du discours qui sera étudié dans la section suivante. Par définition, le rhème, qui regroupe donc rhème et commentaire, correspond à ce qui est dit du thème.

Il existe plusieurs façons de caractériser le thème et le rhème d'un énoncé. Hockett cité dans (Lyons, 1980, p. 133) considère que le thème correspond au sujet grammatical, mais au niveau des référents et non des formes linguistiques. Pour sa part, (Halliday, 1967) fait de la position initiale une condition nécessaire du statut thématique. Dans l'exemple (29) facilement transposable au français, il considère ainsi que le thème est John dans (29a) et yesterday dans (29b). Ce point de vue correspond à celui avec lequel nous avons étudié les 
constructions à topique dans les facteurs liés à l'ordre des mots (et à la saillance de la place initiale), le mot topique s'appliquant au niveau de l'énoncé comme nous le faisons actuellement. Cette condition de place initiale est également confirmée par (Wolters, 2001, p. 44) : "The theme of a clause consists of the constituent(s) which come(s) first in the clause. [...] What comes first in a clause is that is processed first. [...] What comes first influences how the rest is interpreted; this intuition is covered superbly by the way theme is realised in English. ». Une autre considération est également fréquente : le fait que le thème correspond à ce qui est connu de l'interlocuteur et le rhème à qui est nouveau (nous étudierons les saillances relatives du connu et du nouveau dans la section portant sur la saillance liée à des inférences). Thème et rhème sont ainsi définis à l'aide de critères que nous avons déjà étudiés, sans jamais être réduits à l'un de ces critères. Le thème correspond généralement au sujet grammatical, à l'élément placé en premier dans l'énoncé, et à ce qui est connu. Le rhème correspond généralement au prédicat et à ce qui est nouveau. S'il n'y a aucun lien définitif entre thème et sujet grammatical, il semble par contre que le lien entre thème et position initiale soit fort, favorisant ainsi la saillance du thème.

(29) a. John saw the play yesterday

b. Yesterday John saw the play

Dans ces conditions, il est difficile de parler de critère propre au statut de thème. En effet, nous prenons en compte par ailleurs la saillance de l'élément placé en premier, celle du sujet grammatical, ainsi que celle relative à la distinction entre connu et nouveau. Les saillances du thème et du rhème dépendent de tous ces aspects, et ne se définissent pas intrinsèquement. La synthèse de (Caron, 1989) va d'ailleurs dans ce sens. Selon lui, la séquence la plus naturelle de deux phrases est celle où le rhème de la première est repris comme thème de la seconde. Le rhème est repris et en devient donc saillant (c'est le facteur de répétition d'un élément). Quant au thème, les considérations précédentes font qu'il est également saillant. Caron en conclut qu'il est impossible de savoir d'une manière générale si c'est le thème ou le rhème qui est l'élément le plus saillant d'un énoncé. Nous en concluons pour notre part que les notions de thème et de rhème ne possèdent pas de critère intrinsèque de saillance. Ces notions se définissent à l'aide de paramètres que nous avons déjà pris en compte dans notre classification, et rien ne nous pousse à considérer le thème ou le rhème comme un facteur supplémentaire de saillance. Nous décidons en conséquence de ne pas tenir compte de ce facteur, en supposant que d'autres facteurs joueront sans doute pour favoriser la saillance du thème.

\subsection{La saillance liée au propos de la conversation}

44 Au niveau de la sémantique de la conversation ou du texte écrit, le topique ou propos est l'entité dont il est question, qui est discutée ou développée. Pour ne pas faire de mélange avec le topique dont nous avons parlé à la section précédente, nous parlerons ici de propos. Le concept est vague et a donné lieu à plusieurs formulations. Nous noterons en particulier que le propos, bien qu'implicite et intuitif, s'identifie sans problème au cours d'une conversation ou à la lecture d'un texte. Le propos est ainsi l'entité qui matérialise la cohérence d'un dialogue ou d'un texte.

De ce fait, le propos peut recouvrir plusieurs entités du discours. (Wolters, 2001, p. 52) évoque par exemple les structures complexes de van Dijk, avec ses macrostructures et ses superstructures, ainsi que celles de Chafe («aggregates of coherently related events, 
states, and referents »), jusqu'aux termes de supertopic et subtopic qui dénotent la hiérarchisation des éléments de ces structures. M. Wolters compare sur ce point la structure hiérarchique du propos avec la structure d'un document en sections et soussections. S'il peut recouvrir plusieurs entités du discours, le propos peut également correspondre à une entité qui n'est pas mentionnée explicitement. Cette considération met l'accent sur le fait que l'identification du propos d'un texte nécessite l'interprétation complète de ce texte. C'est ainsi que le but, et éventuellement la structure en termes de plans ou de scripts, doivent être pris en compte. Nous en reparlerons dans la section 8.

Face à cette complexité, il s'avère difficile d'attribuer une saillance à d'éventuelles entités $\mathrm{du}$ discours concernées par le propos. Même s'il est clair que le propos est particulièrement saillant, même si l'intuition de n'importe quel locuteur permet d'identifier ce propos et les entités correspondantes, il s'avère difficile de construire un modèle sur cette intuition. Nous avons au contraire besoin de critères formels. Les macrorègles de T. van Dijk et le critère avancé par (Wolters, 2001, p. 49), c'est-à-dire le fait que le propos tend à être introduit par un groupe nominal long et explicite qui n'a pas la fonction sujet, constituent de tels critères. Reste à les regrouper dans un modèle qui puisse aboutir à une formalisation, travail qui dépasse l'objet de cet article et que nous reléguons dans nos perspectives.

\subsection{La saillance liée à des inférences}

Nous nous plaçons ici à un niveau pragmatique, celui de la distinction entre connu (ou donné) et nouveau, et de celle entre posé et présupposé, c'est-à-dire du point de vue de l'information qu'apporte un énoncé par rapport à ce qui est établi. Cette notion d'informativité se rapproche des maximes de (Grice, 1975) et des principes de la Théorie de la Pertinence (Sperber \& Wilson, 1995).

Comme nous l'avons dit plus haut, la distinction entre connu et nouveau est liée à celle entre thème et rhème : du point de vue de l'information apportée, c'est le rhème, donc le commentaire fait sur le thème, qui est nouveau. Elle est également liée à l'accentuation : le nouveau est généralement accentué, et pas le connu (du moins en anglais). Mais ces facteurs ont déjà été discutés, et, pour déterminer l'élément saillant entre connu et nouveau, il nous faut nous intéresser à des critères propres au fait d'être connu, c'est-àdire présent dans la mémoire de l'interlocuteur ou du lecteur, et à celui d'être nouveau, donc d'orienter le discours dans une nouvelle voie. Il est a priori difficile de favoriser l'un ou l'autre: le connu est saillant parce qu'il est cognitivement activé, et parce qu'il est dans une certaine mesure stabilisé ; le nouveau est saillant parce qu'il est susceptible d'intriguer, d'attiser l'intérêt, de focaliser l'attention. (Lyons, 1980) favorise clairement le connu: « ce qui est connu est, pratiquement par définition, plus saillant que ce qui est inconnu; et, toutes choses étant égales par ailleurs, plus une chose a été mentionnée récemment et introduite dans l'univers du discours, plus elle sera familière aux participants et plus elle prédominera du point de vue psychologique » (p. 141). Le critère précis qu'est l'existence d'une ou de plusieurs mentions préalables nous semble devoir être pris en compte. Il diffère de la répétition car il se place à un niveau pragmatique : il peut s'agir de la mention répétée d'une entité du discours à l'aide d'expressions différentes, donc non répétitives. C'est tout l'intérêt des chaînes de référence. Nous garderons ce critère et nous suivrons donc le point de vue de Lyons en attribuant une 
meilleure saillance au connu qu'au nouveau. Les aspects purement cognitifs seront pour leur part débattus plus loin.

La distinction entre posé et présupposé fait intervenir des propositions et pas seulement des entités du discours. Dans l'exemple (30), il est présupposé d'une part que Jacques est marié, d'autre part qu'il battait sa femme. Chacune de ces propositions est plus ou moins saillante : la proposition « Jacques battait sa femme » étant liée au verbe principal de (30) alors que «Jacques est marié » ne reposant que sur le possessif sa, nous pouvons considérer que cette deuxième proposition est moins saillante. Par conséquent, nous pouvons en déduire que l'entité du discours derrière sa femme est moins saillante que celle derrière battre. Cette hiérarchie entre deux présupposés se complète avec le posé qui prend la première place. En effet, c'est avant tout le fait d'arrêter de battre qui est saillant.

(30) Jacques a arrêté de battre sa femme

\subsection{La saillance indirecte par transfert sémantique de saillance}

Pour conclure rapidement sur les aspects sémantiques et pragmatiques, nous noterons, comme nous l'avons fait dans les facteurs grammaticaux de saillance, qu'une entité du discours liée sémantiquement à l'entité identifiée comme étant la plus saillante voit sa saillance augmenter par transfert. Par exemple, si un meurtre est évoqué dans le texte ou dans la conversation, l'entité du discours liée au meurtrier, qu'elle soit évoquée ou non, voit sa saillance augmenter. Dans (Garrod \& Sanford, 1988) que nous avons déjà cité à propos de la saillance des noms propres, on trouve une considération similaire à propos des personnages dans les textes narratifs: les auteurs considèrent que lorsque le personnage principal saillant interagit avec un personnage secondaire, celui-ci en acquiert une certaine saillance par transfert. Nous n'irons pas plus loin dans cette voie, car elle risque de rendre saillantes toutes les entités du discours.

\section{Saillance linguistique : facteurs cognitifs}

51 Nous regroupons ici les facteurs liés au participant, qu'ils correspondent à des processus communs à tout le monde (tels que les processus cognitifs inférieurs: intention, attention, mémoire), ou valables uniquement pour un individu ou pour les membres d'une culture particulière (tels que les facteurs liés à des expériences ou à des connaissances précises).

\subsection{La saillance liée à l'intention}

Un aspect de l'intention correspond à la volonté du sujet de comprendre quelque chose au moment de la lecture ou, dans une discussion, avant même que l'interlocuteur n'ait parlé. Ce phénomène n'est pas si rare. Il est lié d'une certaine façon à l'existence de schémas (ou de scripts): dans un contexte qui comprend une suite d'événements, il arrive que l'événement à venir soit quasiment prévisible compte tenu de la tâche en cours. Même si la suite du texte ou de la discussion ne lui correspond pas, cet événement peut être tellement saillant que le sujet veut et croit le percevoir. Un modèle de saillance intégrant l'intention du sujet doit donc inclure un modèle de succession des événements, et par transitivité des entités de discours liées à ces événements. 


\subsection{La saillance liée à l'attention}

53 d'audition vers des textes ou des paroles. Il s'agit principalement du phénomène de la « soirée cocktail », qui est décrit de manière synthétique dans (Camus et al., 2003) en ces termes: «il correspond à l'incapacité d'un auditeur à comprendre simultanément la totalité des nombreuses conversations qui se déroulent dans une large assemblée. Mais si l'auditeur focalise son attention sur l'une d'entre elles celle-ci devient instantanément intelligible et les autres ne constituent plus alors qu'un confus brouhaha» (p. 45). C'est dans ce sens que l'on peut parler de saillance liée à l'attention, bien que les deux notions s'opposent, comme nous l'avons vu dans la section relative à nos positionnements théoriques.

\subsection{La saillance liée à la mémoire à court terme}

existe des liens très forts entre la saillance et la mémoire à court terme. Ces liens ont pour noms activation, accessibilité, familiarité immédiate, ou encore récence de traitement. Un travail précurseur dans ce domaine est sans doute celui présenté dans (Prince, 1981), décrivant une liste de statuts cognitifs pour des référents linguistiques. Sa hiérarchie inclut ainsi les statuts suivants : non ancré; ancré; inférable à partir de connaissances conceptuelles; inférable à partir de connaissances discursives; évoqué dans le discours; évoqué dans la situation. A chaque fois, son modèle décrit l'identification du statut par une opération s'effectuant sur des représentations mentales.

En partant cette fois des expressions référentielles, (Ariel, 1988) propose une hiérarchie d'accessibilité, c'est-à-dire une liste de rapports entre des formes linguistiques et des niveaux d'accessibilité cognitive. De la plus forte accessibilité à la plus faible, et par conséquent de la plus forte saillance à la plus faible, sa hiérarchie comprend: les marqueurs d'accessibilité extrêmement forte (ellisions, pronoms réfléchis); les pronoms clitiques; les pronoms non accentués; les pronoms accentués; les pronoms accentués accompagnés de gestes ostensifs; les groupes nominaux incluant le démonstratif proximal (cela); les groupes nominaux incluant le démonstratif distal (ceci); les associations du démonstratif proximal avec un modifieur; les associations du démonstratif distal avec un modifieur ; les prénoms ; les noms de famille ; les descriptions définies courtes; les descriptions définies longues; les noms propres; les noms propres complets accompagnés de modifieurs.

ans un même ordre d'idée, (Gundel et al., 1993) propose une échelle de liens entre déterminants et statuts cognitifs. De la saillance la plus forte à la plus faible, cette échelle comprend les statuts cognitifs suivants (les expressions référentielles possibles en anglais apparaissant entre parenthèses) : focalisé (it) ; activé (that, this, this $N$ ) ; familier (that $N$ ) ; identifiable de façon unique (the $N$ ); référentiel (this $N, a N$ ); et un dernier statut correspondant à la possibilité d'identifier un type $(a \mathrm{~N})$.

Toutes ces hiérarchies sont très critiquables. Notre approche fondée sur une caractérisation indépendante des facteurs physiques et des facteurs cognitifs en est complètement opposée. Si les catégories de Prince nous semblent bien correspondre à des facteurs de saillance cognitive (malheureusement assez flous), nous ne suivons pas du tout Ariel et Gundel dans leurs rapprochements entre formes linguistiques et statuts 
cognitifs. Nous sommes en cela de l'avis de Reboul dans sa critique de l'échelle d'Ariel (Reboul \& Moeschler, 1998, p. 130). D'une manière générale, il nous semble qu'il est illusoire d'identifier automatiquement des facteurs cognitifs aussi précis à partir de formes linguistiques. Les contenus de nos sections relatives aux facteurs cognitifs le montrent clairement.

Plutôt que de prendre en compte d'hypothétiques liens entre forme et cognition, nous préférons nous limiter à des critères purement cognitifs tels que celui de (Miller, 1956) sur la taille de la mémoire à court terme, limitée selon l'auteur à sept éléments. Des travaux plus récents, cités dans (Camus et al., 2003), montrent que la limite se situe plutôt à cinq voire à quatre éléments. L'intérêt pour notre propos est de limiter la capacité de notre modélisation de la saillance à sept (ou quatre) entités du discours.

\subsection{La saillance liée à la mémoire à long terme}

Chaque individu apprend les sens des mots de sa langue à travers des expériences qui lui sont propres et qui connotent peu à peu ces sens. Dans la mémoire à long terme sont ainsi associées différentes connotations à chaque mot, à chaque concept. Certaines associations peuvent être très fortes pour un individu, et nous pouvons parler en ce sens de saillance par familiarité individuelle. Un aspect de la saillance liée à la mémoire porte aussi sur la connaissance durable et culturelle d'un prototype. C'est le facteur entrenchment de (Pattabhiraman, 1993, p. 36), facteur dénotant le fait qu'une propriété peut se figer peu à peu, et ainsi devenir saillante par stabilisation.

Un individu appartient de plus à une culture, et apprend à se familiariser avec certaines caractéristiques propres à cette culture. La lecture de gauche à droite ou de droite à gauche en est un exemple classique qui peut avoir des répercussions sur la saillance des éléments d'un texte écrit. D'une manière générale, des hiérarchies de saillance se mettent en place lors des interactions entre les différents membres d'une communauté, et nous pouvons parler en ce sens de saillance par familiarité culturelle.

\subsection{La saillance liée à la personnalité}

61 Chaque individu se caractérise bien entendu par sa personnalité, son caractère. Nous n'explorerons pas cette voie très vaste et nous resterons dans le domaine des travaux touchant à la saillance dans un contexte linguistique. Ainsi, nous citerons à la suite de (Pattabhiraman, 1993, p. 37) la saillance liée à l'ego du sujet, avec le principe du «moi en premier » de Cooper \& Ross. Les conséquences de ce principe sont une mise en avant des caractéristiques de la situation immédiate du sujet: premièrement ses coordonnées spatiales immédiates, révélées par exemple par le déictique pur ici; deuxièmement sa propre personne révélée par des expressions telles que celle de l'exemple (31); et troisièmement ses affaires et ses problèmes qu'il met souvent en avant, ce qui favorise une tournure telle que (32a) plutôt que (32b). Ces considérations rejoignent le critère de saillance de speaker motivation de (Osgood \& Bock, 1977), critère qui est d'ailleurs appuyé par les auteurs à l'aide de l'exemple (32).

(31) Moi, je pense que...

(32) a. My car was stolen

b. Someone stole my car 


\subsection{La saillance liée à l'affect et aux émotions}

62 En ce qui concerne l'affect et les émotions, (Pattabhiraman, 1993, p. 27) parle de vividness pour décrire la capacité d'une entité du discours à évoquer des représentations émotives et affectives. Il donne ainsi l'exemple très évocateur du mot vampire. Ce facteur de saillance joue également pour convaincre un interlocuteur, par exemple dans les slogans de publicités. L'auteur définit également la notion très proche de imageability, qui correspond à la capacité à évoquer des représentations mentales claires et sans ambiguïté (comme pour un triangle), ainsi que la notion de concreteness, qui dans un même ordre d'idée renforce la saillance des objets concrets par rapport aux concepts abstraits.

La saillance liée à l'affect concerne surtout les mots et les sons qui sont dotés d'une signification particulière pour l'individu. C'est le cas de son propre nom, de celui de la personne qu'il aime, des pleurs de son enfant, etc. Le nom de l'individu est cité par (Camus et al., 2003, p. 46) dans sa présentation du phénomène de la "soirée cocktail » dont nous avons parlé dans les facteurs cognitifs: «si dans un groupe voisin le nom de l'auditeur est prononcé, ce dernier ne va pas pouvoir s'empêcher de tendre l'oreille pour écouter ce qui se raconte à son propos». Cette considération au niveau des mots peut bien sûr être étendue au niveau des entités et des propositions portées par les énoncés.

En distinguant ici aussi facteurs individuels et facteurs culturels, un dernier exemple important pour la notion de saillance concerne les sujets culturellement saillants pour l'affect, par exemple tout ce qui concerne la mort ou la souffrance, et, comme le rappelle (Rousselet \& Fabre-Thorpe, 2003) à propos de saillance visuelle, le sexe. Les mots relatifs à ces sujets, avec les connotations qu'ils ont acquises, se voient attribuer une saillance qui fait désormais quasiment partie d'eux-mêmes, leur donnant presque le statut de déclencheurs automatiques d'émotions.

\section{Saillance visuelle : facteurs physiques}

65 Après la saillance linguistique, étudions maintenant la saillance visuelle, en tentant la même séparation entre facteurs physiques et facteurs cognitifs. Liés aux particularités de la perception visuelle, les facteurs physiques par lesquels nous commençons dans cette section sont a priori très différents des facteurs physiques de saillance linguistique.

\subsection{La saillance liée aux contrastes de luminosité et de couleur}

Un premier facteur est la saillance intrinsèque à la nature de l'objet en terme de luminosité. Il s'agit par exemple de la saillance du soleil ou d'une ampoule électrique du fait de leurs luminosités inhérentes.

Deuxième facteur que nous proposons, la saillance liée aux variations de perception des couleurs recouvre deux aspects : un premier relatif aux temps de latence, et un second relatif à l'effet spatial des couleurs. Le temps de latence, c'est-à-dire le décalage entre le début de l'excitation et celui de la sensation, varie selon les couleurs. Baticle (1985) donne les chiffres suivants : rouge (22.6 millièmes de seconde); vert ( $37.1 \mathrm{~ms})$; gris (43.4 ms); bleu $(59.8 \mathrm{~ms})$; jaune $(96.3 \mathrm{~ms})$. Ces chiffres qui constituent une échelle de saillance doivent néanmoins être relativisés : pendant le jour, le maximum de sensibilité est dans le jaune et le rouge, alors que c'est le bleu le plus saillant pendant la nuit. D'autre part, 
comme le montrent les photographies de (Freeman, 1989) et les exercices de (Itten, 1985), une couleur appelle sa complémentaire. Un objet qui prend la couleur complémentaire de celle $d u$ fond en devient par exemple très saillant. Plus généralement, la saillance de chaque couleur dépend de son association avec d'autres couleurs. (Cocula \& Peyroutet, 1986) propose ainsi une hiérarchie de couples de couleurs en fonction de l'importance de leur impact visuel : noir sur blanc; puis noir sur jaune ; puis rouge sur blanc; etc. Enfin, (Itten, 1985) note l'importance de l'effet spatial des couleurs : contrairement à du violet, du jaune sur fond noir a l'air d'avancer et voit ainsi sa saillance augmenter. Ces données nous donnent les bases pour un modèle de la saillance chromatique d'un objet en fonction de la luminosité ambiante, de la couleur dominante du fond et de la couleur dominante de l'objet.

\subsection{La saillance liée à une singularité dans un ensemble d'objets}

Il s'agit ici de la saillance due à une mise en relief contextuelle du fait de la présence de plusieurs objets. Compte tenu d'un ensemble d'objets, un élément de cet ensemble est saillant s'il se distingue par une propriété que les autres éléments n'ont pas. Autrement dit, être un singleton est un facteur de saillance, et, à l'exception des deux facteurs évoqués dans la section précédente, la saillance ne découle pas d'une propriété particulière, mais du fait d'être un singleton pour une propriété. (Edmonds ,1993) donne ainsi l'exemple d'un immeuble a priori saillant par sa grande taille, et qui perd toute saillance lorsqu'il est entouré d'immeubles encore plus grands. En déroulant les types de propriétés d'un objet physique, nous pouvons parler de saillance liée à la catégorie des objets, de saillance liée aux caractéristiques physiques que sont la forme, la couleur, la taille, le matériau ou la texture, de saillance liée à l'orientation de l'objet, et de saillance liée à sa dynamique. Nous considérons qu'il existe une hiérarchie des propriétés qui correspond à l'ordre dans lequel nous les avons citées. La primauté de la catégorie est démontrée par la fréquente nécessité d'utiliser un substantif catégorisant dans une expression référentielle. Si une chaise rouge se distingue dans une scène, on dira plus facilement la chaise ou la chaise rouge que l'objet rouge, le terme objet jouant ici le rôle de substantif non catégorisant. En ce qui concerne l'importance relative des propriétés que sont la forme, la couleur et la taille, nous donnons la primauté à la forme car elle correspond souvent à la propriété fondamentale de l'objet. Un exemple de (Pattabhiraman, 1993) le montre clairement dans le cas de comparaisons et de métaphores: dans les joues de Mary sont comme des pommes, sauf qu'elles sont jaunes, la comparaison se fait implicitement sur la forme ronde. L'inversion de l'ordre d'importance de la forme et de la couleur aboutit à un exemple déconcertant: les joues de Mary sont comme des bananes, sauf qu'elles sont rondes. Entre couleur et taille, des expérimentations avec mesure des temps de réponse montrent que la couleur est traitée plus rapidement que la taille et intervient avant elle dans le processus de perception (Baticle, 1985). En génération automatique, (Reiter \& Dale, 1997) va dans le même sens en privilégiant la production de l'expression référentielle le chien noir plutôt que le petit chien, dans une scène confrontant un grand chien blanc et un petit chien noir. En ce qui concerne les saillances liée à l'orientation et à la dynamique, les exemples suivants montrent clairement que le fait d'être un singleton pour l'une de ces propriétés est un facteur de saillance : dans une rangée de chaises, une orientation différente suffit à rendre saillante la chaise incriminée; dans une scène contenant un objet animé et plusieurs objets 
inanimés, l'objet animé est saillant ; dans une scène contenant un objet en mouvement et plusieurs objets statiques, l'objet en mouvement est saillant.

Si nous considérons maintenant que des objets dans une scène peuvent se regrouper pour former des groupes perceptifs qui sont perçus en tant qu'amas d'objets avant d'être perçus en tant qu'objets individuels, nous pouvons parler de saillance liée à l'isolement ou à l'appartenance à un groupe perceptif. Ce facteur peut se modéliser en exploitant les principaux critères de la Théorie de la Gestalt que sont la similarité, la proximité et la bonne continuité (Wertheimer, 1923). En regroupant les objets proches et ayant des formes et des couleurs similaires, ou en regroupant des objets similaires qui forment des lignes, il est possible de structurer une scène en groupes perceptifs, et d'appliquer la saillance à ces groupes. Ainsi, un objet isolé est particulièrement saillant si tous les autres objets visibles appartiennent à des groupes perceptifs (il s'agit ici aussi de singleton).

\subsection{La saillance liée à la cohésion et la structuration de la scène}

70 Un premier facteur dans cette catégorie est la saillance due à la mise en évidence explicite d'un objet dans la scène. Cette mise en évidence peut être due à un éclairage particulier, par exemple dans le cas d'un comédien éclairé par des spots, ou par un contraste figure sur fond important. Dans le cadre de la communication homme-machine, elle peut également provenir d'une intention ostensive de la part du système, avec l'utilisation d'un rendu visuel particulier (textures transparentes, affichage de la boîte englobante de l'objet), ou d'une intention ostensive de la part de l'utilisateur, lorsque celui-ci produit un geste de pointage qui rend saillant l'objet désigné.

71 Un deuxième facteur très proche mais plus implicite est la saillance due à une construction dédiée. C'est le cas du sujet d'une photographie quand celle-ci est composée de manière à valoriser ce sujet, par exemple à l'aide d'une construction en triangle qui, plaçant le sujet au sommet supérieur, le rend saillant (Freeman, 1989).

72 Vient ensuite la saillance due au placement à un endroit stratégique. Ce facteur fait intervenir la notion de cadre de l'image et de point fort compte tenu du cadre. Toute image se caractérise par un cadre, correspondant par exemple à un rapport 16/9 ou 4/3 comme c'est le cas d'un écran d'ordinateur. Dans ce cadre, le centre est un point fort immédiat, c'est-à-dire qu'il attire le regard en priorité. Les autres points forts sont les intersections des lignes horizontales et verticales situées aux tiers du cadre, ou des lignes correspondant à la « divine » proportion du nombre d'or (Cleyet-Michaud, 1973). Un objet placé à l'un de ces points est potentiellement saillant. Cette saillance est d'autant plus forte que le cadre est marqué. (Freeman, 1989) montre ainsi des exemples de cadres imbriqués qui rendent particulièrement saillant l'objet placé en leur centre. Certains points forts dépendent non pas du cadre mais de l'organisation des objets présents dans la scène. Il s'agit par exemple des zones situées dans le prolongement ou à l'intersection des lignes de force qui structurent la scène et dirigent le regard de l'observateur.

73 Nous regroupons dans un quatrième facteur les saillances liées à la perspective et aux équilibres. La première consiste à privilégier le ou les points de fuite lorsque la perspective est marquée; la seconde consiste à privilégier le point d'équilibre lorsque l'image présente un équilibre des masses qui repose sur ce point (Sanmiguel, 2000). Encore une fois, tout objet placé en l'un de ces points est potentiellement plus saillant qu'un objet placé ailleurs. 
74 La saillance liée aux répétitions et aux symétries constitue un cinquième facteur. Plusieurs objets identiques, surtout s'ils sont placés de manière régulière, induisent une répétition insistante qui les rend saillants. Dans un même ordre d'idée, plusieurs objets placés de manière symétrique entraînent une perception particulière de ces objets ainsi que de celui placé au niveau du centre de symétrie. Autre exemple, des lignes en zigzag font ressortir les objets situés aux cassures des lignes.

Un dernier facteur est la saillance indirecte par transfert visuel de saillance, c'est-à-dire la saillance s'appliquant à un objet qui est relié à un objet fortement saillant, le lien étant dû à une proximité particulière ou découlant des lignes de force qui dirigent le regard de l'observateur compte tenu de la structure de l'image. Ce facteur s'avère difficile à modéliser, d'une part car l'identification de lignes de force est un problème complexe (Vettraino-Soulard, 1993), et d'autre part car le principe de la transitivité tend à rendre saillants tous les objets de la scène et à enlever tout intérêt à la notion de saillance.

\section{Saillance visuelle : facteurs cognitifs}

Pour la perception visuelle comme pour le langage, chacun des aspects cognitifs que sont l'intention, l'attention, la mémoire, ou encore l'affect, sont liés à la saillance. Nous retrouvons ainsi dans cette section quasiment la même répartition en sous-sections que dans la section 5 .

\subsection{La saillance liée à l'intention}

Un premier facteur cognitif de saillance est ainsi la saillance liée à l'intention, c'est-à-dire à l'influence de la tâche en cours sur la perception. A la suite de son exemple sur les propriétés saillantes d'immeubles, (Edmonds, 1993) souligne que certaines propriétés sont saillantes dans un but précis et pas dans un autre but. Dans son contexte de description d'un itinéraire routier, la propriété de taille est ainsi saillante lorsque la tâche est la désignation d'un immeuble, mais ne l'est pas lorsqu'il s'agit de désigner une intersection de rues. Dans ce sens, un modèle de saillance requiert un modèle de la tâche. La saillance liée à l'intention recouvre également deux autres aspects : les fonctionnalités des objets et leur incongruité compte tenu d'une intention. Les fonctionnalités des objets peuvent être perçues visuellement, et concourir ainsi à la saillance. C'est le cas, pour un utilisateur potentiel, d'un ordinateur allumé dans une pièce contenant d'autres ordinateurs éteints. En ce qui concerne l'incongruité ou l'aspect énigmatique d'un objet compte tenu d'une intention, nous noterons qu'un objet dans une situation incongrue est en infraction avec une règle implicite, culturelle ou fonctionnelle, et acquiert de cette infraction une certaine saillance. C'est le cas d'une chaise renversée ou placée sur une table lorsqu'on a l'intention de s'asseoir, ou d'une chaise placée en plein milieu de la pièce dans laquelle on fait le ménage. De même, une casserole est a priori plus saillante dans une salle de bain que dans une cuisine (sauf si elle sert à contenir une fuite d'eau, c'est-à-dire si sa fonction prend le pas sur l'incongruité). Plus particulièrement sur l'aspect énigmatique, nous retiendrons qu'un objet qui n'est pas à sa place compte tenu de la tâche en devient saillant, de même qu'un objet inattendu. Ce dernier exemple se rapproche de l'effet de nouveauté, ou unexpectedness de (Conklin \& McDonald, 1982). 


\subsection{La saillance liée à l'attention}

78 Un deuxième facteur cognitif est la saillance liée à l'attention visuelle, dans le sens de focalisation à un sous-espace visuel ou de meilleure détection des stimuli du fait d'une attitude de préparation à la perception. L'attention consiste dans ces deux cas en un traitement prioritaire de données perceptives. L'existence de sous-espaces visuels est montrée par (Kessler et al., 1996). Dans leur expérimentation, des sujets sont invités à référer linguistiquement à des objets perçus visuellement et séparés en deux groupes perceptifs distincts. Certaines expressions référentielles produites s'avèrent ambiguës si l'on considère la scène complète, mais non ambiguës si l'on se place dans l'un des groupes perceptifs. Ce résultat montre que les sujets ont procédé à une focalisation attentionnelle, qui les a conduit à ignorer le reste de la scène. Un exemple du deuxième cas est celui de personnes qui entrent dans une pièce et cherchent des chaises pour s'asseoir : avec cette attitude de préparation, les chaises deviennent plus saillantes que les autres meubles. Dans ce contexte, l'effet de nouveauté n'est pas un facteur de saillance, ce qui illustre une différence entre intention et attention.

\subsection{La saillance liée à la mémoire à court terme}

Le troisième facteur cognitif est la saillance liée à la mémoire à court terme, qui concerne les objets récemment traités par le système cognitif visuel, et se rapproche du facteur de vécu récent de (Rousselet \& Fabre-Thorpe, 2003). Le principal critère pour une modélisation de ces aspects est la capacité limitée à sept éléments (Miller, 1956), capacité revue à cinq ou quatre éléments dans des travaux plus récents (cités par Rousselet et Fabre-Thorpe). Reste à déterminer quels sont les quatre ou cinq éléments visuels qui seront retenus. La récence de traitement n'est en effet par le seul critère, comme le montre (Pylyshyn, 2001) avec la notion d'index visuel. Un index est une sorte d'étiquette ou de fichier. Lié à un élément visuel et destiné à le repérer rapidement (sans le décrire), il ne s'applique qu'aux éléments saillants d'une scène, ceux qui seront traités et retenus de manière prioritaire. Z. Pylyshyn décrit le fonctionnement de ces index, et nous pouvons considérer à sa suite que les quatre ou cinq derniers objets repérés par un index sont cognitivement plus saillants que les autres objets visuels.

\subsection{La saillance liée à la mémoire à long terme}

Quatrième facteur, la saillance liée à la mémoire à long terme peut se décomposer en deux aspects, premièrement la familiarité visuelle individuelle, et deuxièmement la familiarité visuelle culturelle. Un exemple de familiarité individuelle est celui de l'infographiste habitué à travailler sur des images et à corriger leurs couleurs : pour lui, un contraste entre deux teintes de rouge sera saillant, alors que le même contraste pourra ne pas être perçu par un autre individu. Plus généralement, nous acquérons tous nos propres sensibilités à certaines couleurs (il n'est pas nécessaire d'être daltonien), nos propres critères de saillance. Dans le cadre de la communication homme-machine, un modèle de saillance requiert dans ce sens un modèle de l'utilisateur. Un exemple de familiarité culturelle est le fait que, dans notre vie en société, nous avons l'habitude de communiquer avec nos semblables. Il est ainsi possible de classer selon leur saillance les participants d'une situation visuelle : la présence d'un être humain dans notre champ de 
vision est saillante avant tout (surtout s'il s'agit de quelqu'un que l'on connaît); vient ensuite la saillance d'un animal ; puis celle des objets inanimés. Un autre exemple relatif à une communauté est celui des couleurs du drapeau national. (Rastier, 1991) note que les couleurs de son propre pays sont culturellement saillantes pour un individu. (Segall et al., 1966) montre de manière détaillée comment la perception visuelle varie selon les cultures. Avec comme base d'expérimentation certaines illusions visuelles classiques, ils distinguent par exemple les cultures « circulaires » habituées aux courbes et peu affectées par les illusions mettant en jeu des droites, et les cultures comme la nôtre où les lignes droites et parallèles sont fréquentes (rues, immeubles, etc.). Plus généralement, l'appartenance d'un individu à une communauté, quelle qu'elle soit, entraîne la possibilité de facteurs spécifiques de saillance.

\subsection{La saillance liée à l'affect et aux émotions}

81 Tout stimulus visuel peut en effet provoquer chez un individu particulier certaines émotions. (Pattabhiraman, 1993) parle ainsi de vividness pour décrire la capacité d'un stimulus à évoquer des représentations émotives, et donne l'exemple d'un vampire. Certains objets acquièrent ainsi une forte connotation émotionnelle. Comme le rappelle (Rousselet \& Fabre-Thorpe, 2003), c'est particulièrement le cas des stimuli sexuels. De même, la perception du visage d'un interlocuteur peut conduire à l'identification immédiate de ce que ressent cet interlocuteur : à partir de l'expression la plus discrète, du stimulus le plus minime, on est capable de discerner aisément des sentiments tels que la peur, l'amour, la passion, le stress, ou encore le dégoût.

\section{Bilan : saillance physique et saillance cognitive}

Notre inventaire des facteurs de saillance peut-il constituer une base pour une caractérisation générique de la notion de saillance? Maintenant que nous avons une idée précise des facteurs de saillance, il est temps de souligner les parallèles entre les aspects linguistiques et les aspects perceptifs, afin d'en déduire un ensemble de facteurs génériques de saillance. Cet ensemble que nous décrirons dans une première sous-section nous semble constituer un premier pas vers une future modélisation de la notion de saillance. Dans une deuxième sous-section, nous remettrons en question la distinction entre saillance physique et saillance cognitive, avec un certain nombre d'arguments qui seront autant de perspectives de recherche. Enfin, nous poserons la question de l'existence de plusieurs niveaux de traitement de la saillance, et nous conclurons sur l'intérêt d'un système d'identification automatique d'entités saillantes.

\subsection{Vers une caractérisation générique de la notion de saillance}

83 Nous avons étudié séparément la saillance linguistique puis la saillance visuelle, et nous pouvons remarquer un certain nombre de similitudes entre ces études. Nous l'avions noté avec les facteurs cognitifs, qui peuvent se classer selon les mêmes catégories dans une modalité ou dans l'autre. Comme le montre le tableau 1, c'est également le cas des facteurs physiques : nous retrouvons des catégories similaires entre saillance linguistique et visuelle, avec des exemples qui se rapprochent aisément. 
84 saillance intrinsèque à la nature de l'objet. Que l'unité soit visuelle ou linguistique, il est dans les deux cas possible de parler de critère intrinsèque : un exemple visuel est la luminosité inhérente au soleil, et un exemple linguistique est la saillance que possède intrinsèquement un nom propre. La simplicité de l'unité constitue également un facteur générique de saillance : un objet qui se rapproche de la notion de bonne forme (dans le sens de la Théorie de la Gestalt) et un mot tel qu'une interjection composée d'une seule syllabe sont ainsi saillants par nature. Nous avons vu à quel point le fait d'être un singleton pour une propriété rend une unité saillante. Ce constat s'avère également valable en linguistique: lorsqu'un seul antécédent est possible, sa saillance facilite d'autant l'interprétation d'un pronom anaphorique. Isoler une unité linguistique peut se faire à l'aide d'une apposition (ou d'une parenthèse). L'accentuation sert à mettre explicitement en évidence un mot ou un groupe de mots. Quant au présentatif, il est dédié à la saillance de l'entité du discours qu'il met en avant, par exemple du triangle rouge dans c'est le triangle rouge qu'il faut supprimer. Nous retrouvons donc aussi bien dans le message linguistique que dans le message visuel le facteur consistant à placer une unité à un endroit stratégique pour la rendre saillante. C'est typiquement le rôle de la position initiale dans un énoncé. Les répétitions et les symétries existent aussi bien dans la perception visuelle que dans le langage. La figure de style qu'est le chiasme, c'est-à-dire une alternance du type A-B-B-A, est un exemple de symétrie linguistique qui rend saillants les composants A et B. Enfin, les facteurs que sont la rupture dans une continuité, l'infraction d'une règle implicite, l'exploitation d'une norme et la structuration du message pour la mise en avant d'un élément, nous semblent particulièrement intéressants en tant que facteurs génériques de saillance.

\begin{tabular}{|l|l|l|}
\hline Facteur générique de saillance & Exemple visuel & Exemple linguistique \\
\hline Critère intrinsèque aux unités & Luminosité inhérente & nom propre \\
Simplicité & Bonne forme & interjection \\
Singleton & Singleton de couleur & seul antécédent \\
Isolement & Isolement spatial & apposition \\
mise en évidence explicite & Eclairage spot & accentuation \\
construction dédiée & Valorisation du sujet & présentatif \\
placement à un endroit stratégique \\
répétition & Tiers du cadre & début de l'énoncé \\
symétrie & Régularité & répétition \\
rupture dans une continuité & Symétrie & chiasme \\
infraction d'une règle implicite & Disposition linéaire & rythme d'élocution incongrue \\
exploitation d'une norme & raté, erreur \\
structuration du message & Ligne de force & construction usuelle \\
construction à topique \\
\hline
\end{tabular}

Tableau 1 : premier pas vers la détermination d'un ensemble de facteurs génériques de saillance.

Bien qu'il ne soit pas encore abouti, l'ensemble de ces exemples montre à quel point la saillance est un phénomène global qui recouvre des disciplines variées. C'est pour cette 
raison qu'il nous semble plus pertinent de distinguer la saillance physique de la saillance cognitive, plutôt que de séparer les définitions et les analyses de la saillance selon la modalité d'étude, visuelle ou linguistique. Comme nous allons maintenant le voir, notre distinction entre les notions de P-saillance et de C-saillance, comme nous les avons nommées dans l'introduction de cet article, reste cependant critiquable.

\subsection{Pertinence de la distinction entre P-saillance et C-saillance}

Nous avons vu que, pour le message linguistique aussi bien que pour le message visuel, il est possible de distinguer d'un côté des facteurs physiques, et d'un autre côté des facteurs cognitifs. La nature de ces facteurs ne varie pas beaucoup, seule leur matérialisation dans la modalité considérée, linguistique ou visuelle, varie. Ceci justifie à nos yeux l'importance de la distinction entre les notions de P-saillance et de C-saillance. Tout au long de notre inventaire, nous avons ainsi tenu à rapprocher les types de facteurs linguistiques avec les types de facteurs visuels. C'est ainsi que l'on retrouve des facteurs liés à l'intention, à l'attention, à la mémoire à court terme, etc., aussi bien pour la saillance linguistique que pour la saillance visuelle.

Pourtant, certains facteurs classés dans les facteurs physiques auraient aussi leur place parmi les facteurs cognitifs. C'est le cas par exemple de la saillance liée au propos de la conversation. Dans la section 4.4, nous avions noté que l'identification du propos nécessite une interprétation complète du texte. Ceci peut amener la prise en compte de la structure intentionnelle, et par conséquent de certains aspects cognitifs. Selon ce point de vue qui consiste à rattacher le propos au locuteur plutôt qu'au discours, la saillance liée au propos serait un facteur cognitif. Avec le même point de vue, la saillance liée au thème de l'énoncé et la saillance liée à des inférences seraient aussi des facteurs cognitifs. Nous considérons pour notre part que ces facteurs ont une matérialisation linguistique suffisamment concrète pour les classer dans les facteurs physiques: ils se déduisent facilement d'un matériau physique, en tout cas beaucoup plus facilement que la mémoire à court terme et les autres facteurs purement cognitifs.

Cette question sur la nature de la saillance liée au thème ou au propos soulève une autre question : y aurait-il parmi les facteurs visuels des équivalents du thème et du propos, et ces équivalents pourraient-ils être classés parmi les facteurs cognitifs? On peut en effet considérer qu'une image de publicité comprend un thème relatif au produit à vendre, ainsi qu'un rhème consistant à donner des arguments visuels pour favoriser la vente. Le produit est particulièrement mis en avant dans l'image, et correspond bien à l'élément saillant. Reste à délimiter cette notion de rhème visuel par rapport aux notions de ligne de force ou de composition. L'image garde son mode de fonctionnement et sa cohérence, et il nous semble un peu prématuré de définir un thème et un propos visuels.

Nous restons donc sur cette distinction entre P-saillance et C-saillance qui peut permettre de mieux appréhender la nature de la saillance. Nous espérons ainsi contribuer à une meilleure prise en compte de cette notion, en particulier dans les travaux portant sur la structure communicative linguistique : il nous semble en effet que celle-ci se caractérise aussi bien par l'ensemble de nos facteurs génériques que par les notions classiques de focus, thème et topique. 


\subsection{Plusieurs plans de traitement de la saillance?}

90 Nous avons considéré dans cet article la saillance comme une notion indépendante du type de traitement réservé aux entités saillantes : que ce soit pour la compréhension ou la production d'un message linguistique, un certain nombre de facteurs concourent à attribuer à une certaine entité de discours le statut d'entité la plus saillante. L'échelle est unique, et, si plusieurs entités peuvent être saillantes à des degrés divers, nous n'avons pas prévu de cas où telle entité serait la plus saillante pour tel traitement et telle autre entité la plus saillante pour tel autre traitement. La question qui se pose maintenant est celle de l'existence de plusieurs plans de traitement qui justifient autant de caractérisations de la saillance. Il ne s'agit cependant pas de remettre en question l'inventaire des facteurs de saillance, mais seulement leur importance relative dans certains contextes.

91 En reprenant certains des critères étudiés, on peut considérer deux composants principaux d'un énoncé : d'une part l'élément mis en avant, qui justifie la construction de la phrase, et d'autre part le contenu informatif, c'est-à-dire ce que l'énoncé apporte de nouveau et de pertinent. Le premier élément correspond souvent au thème; il est souvent déjà connu par l'interlocuteur et sa place naturelle est le début de phrase, avec l'aide d'un présentatif ou non. Il constitue une introduction à ce dont on va parler. Le deuxième élément correspond souvent au rhème et recouvre le reste de la phrase, qui peut comporter bien plus qu'une ou deux entités du discours. Plutôt que de considérer une seule entité saillante aux yeux de l'interlocuteur, on peut distinguer une entité saillante dans le cadre du premier élément, et une entité saillante dans le cadre du deuxième élément. Dans un cas comme dans l'autre, les facteurs de saillance appartiennent à notre classification, même si le premier élément est surtout lié à des facteurs physiques (le deuxième élément faisant intervenir aussi bien des facteurs physiques liés au sens de l'énoncé que des facteurs cognitifs). Cette méthode permet de rendre compte de cas où un énoncé regroupe à la fois une mise en introduction particulièrement forte ainsi qu'une information nouvelle particulièrement importante. Il apparaît pertinent de déterminer une entité saillante dans chacun d'eux. Reste à appliquer et à tester ce principe sur des corpus, ainsi qu'à déterminer l'importance de chaque facteur à l'intérieur de tel ou tel plan. Ces perspectives dépassent elles aussi le cadre de cet article, dont le but principal reste une mise à plat des facteurs de saillance, et leur classification selon leur point d'ancrage, physique ou cognitif.

\section{Conclusion}

92 La saillance ne fait pas explicitement partie du message communiqué, mais tout le message se fonde sur elle, se structure en fonction d'elle, et s'explique par elle. Nous avons voulu ici éclaircir la nature de cette notion qui recouvre de nombreux aspects. Notre approche pluridisciplinaire nous a amené à proposer une classification de facteurs de saillance à la fois large dans les phénomènes couverts et tendant vers un modèle ultérieur plus formel. Parmi les facteurs physiques et les facteurs cognitifs que nous avons détaillés, certains sont d'ores et déjà formalisables. Du côté linguistique, il s'agit en particulier de l'ensemble des facteurs physiques liés à la forme de l'énoncé, et, parmi les facteurs liés à son sens, ceux relatifs à la sémantique des mots ainsi que, dans une 
certaine mesure, ceux relatifs aux rôles thématiques et aux inférences pragmatiques. Du coté visuel, il s'agit globalement des facteurs physiques.

Parmi les difficultés rencontrées, nous noterons que certains facteurs se recoupent, par exemple la construction syntaxique dédiée qui vise à placer une entité du discours en première position (ce qui correspond à un autre facteur), ou encore l'exemple de la notion de topique qui inclut des aspects syntaxiques, pragmatiques et cognitifs. Certains aspects n'ont pas été traités, tels qu'une analyse poussée des interactions possibles entre les différents facteurs, ou encore une discussion sur l'applicabilité de certains facteurs visuels à différents types de scènes. En particulier, nous noterons que les facteurs physiques liés à la cohésion de la scène nous semblent surtout pertinents pour une image structurée, mais pas forcément pour une image tout venant. Cette remarque a son écho en linguistique: certains exemples comme le chiasme interviennent surtout pour du texte travaillé (poésie), et beaucoup moins pour du texte tout venant. Pour aller plus loin sur ces aspects, nous noterons que notre classification pourrait être améliorée par la prise en compte de nouveaux facteurs, en particulier rhétoriques et stylistiques. Ce serait le cas avec la prise en compte de la protase, de l'acmé et de l'apodose (avec l'attribution a priori de la meilleure saillance à l'acmé) pour ce qui concerne la saillance linguistique, et la prise en compte de facteurs esthétiques pour ce qui concerne la saillance visuelle.

Enfin, nous voulons rappeler l'intérêt de disposer d'un modèle de saillance dans les systèmes de compréhension et de génération automatiques. En compréhension, la saillance n'est pas seulement utile à la résolution des anaphores pronominales. Elle sert d'une manière générale à la résolution de la référence, et particulièrement lorsque l'expression est a priori ambiguë: lorsque le système doit interpréter le $N$ dans un contexte comprenant plusieurs objets du type $\mathrm{N}$, que ce contexte soit visuel, linguistique ou lié à la tâche, une prise en compte de la saillance peut permettre de décider d'axer l'interprétation sur le $N$ le plus saillant. C'est par exemple l'approche de (Clark et al., 1983). La saillance a aussi un rôle de prédiction: lorsqu'une entité du discours est saillante, on peut s'attendre à ce que le locuteur va en parler. Cet aspect est d'importance dans les systèmes de dialogues oraux, à la fois pour la phase de reconnaissance et celle de compréhension. Lors de la reconnaissance des mots prononcés par l'utilisateur, il s'agit par exemple d'entraîner des modèles statistiques pour améliorer les performances en prédisant le mot à venir. Lors de la compréhension proprement dite, il s'agit de phénomènes de focalisation sur les aspects contextuels saillants, par exemple par l'intermédiaire de domaines de référence saillants comme nous le faisons dans (Landragin, 2004). En génération automatique, (Pattabhiraman, 1993) montre comment la saillance peut être exploitée à chacune des étapes que sont : la sélection du contenu (le " quoi dire »); son organisation; les choix lexicaux; et la génération d'une structure de surface (le "comment le dire »). Nous ajouterons que la saillance peut également être utile pour la fouille de texte et l'identification de mots-clés, pour le résumé automatique, l'aide à la traduction, et d'une manière générale pour toutes les applications de traitement automatique du langage naturel. Avec un point de vue plus cognitif, la saillance est également d'utilité dans les modèles de communication, en exploitant par exemple l'importance cognitive des informations saillantes (comme le fait la publicité), ainsi que dans les tâches d'apprentissage, car elle peut permettre d'identifier la forme qui rend saillant le savoir à transmettre. C'est dans ce sens qu'il nous semble important de continuer les recherches dans ce domaine. 


\section{BIBLIOGRAPHIE}

Alshawi H. (1987) Memory and Context for Language Interpretation, Cambridge, Cambridge University Press.

Ariel M. (1988) Referring and Accessibility, Journal of Linguistics, 24:1, pp. 65-87.

Baticle Y. (1985) Clés et codes de l'image, Paris, Magnard Université.

Camus J.-F., Drake C., Brochard R. (2003) Les processus attentionnels en audition, Psychologie Française, 48:1, pp. 45-60.

Caron J. (1989) Précis de psycholinguistique, Paris, PUF.

Chafe W. (1994) Discourse, Consciousness, and Time: The Flow and Displacement of Conscious Experience in Speaking and Writing, Chicago, University of Chicago Press.

Clark H. H., Schreuder R., Buttrick S. (1983) Common Ground and the Understanding of Demonstrative Reference, Journal of Verbal Learning and Verbal Behavior, 22, pp. 245-258.

Cleyet-Michaud M. (1973) Le nombre d'or, Paris, PUF (Que sais-je ?).

Cocula B., Peyroutet C. (1986) Sémantique de l'image. Pour une approche méthodique des messages visuels, Paris, Delagrave.

Conklin E. J., McDonald D. D. (1982) Salience: The Key to the Selection Problem in Natural Language Generation, In Proceedings of the 20th Annual Meeting of the Association for Computational Linguistics, Toronto, pp. 129-135.

Cooper W. E., Ross J. R. (1975) World Order, In Papers from the Parasession on Functionalism, Grossman R. E., James San L., Vance T. J. (eds.), Chicago Linguistic Society, pp. 63-111.

Cordier F., François J., Victorri B. (eds.) (2000) Syntaxe et Sémantique, Vol. 2, Caen, Presses Universitaires de Caen.

Cosnier J., Vaysse J. (1997) Sémiotique des gestes communicatifs, Nouveaux actes sémiotiques, 52-53-54, pp. 7-28.

Edmonds P. G. (1993) A Computational Model of Collaboration on Reference in Direction-Giving Dialogues, Ms. Thesis, University of Toronto.

Freeman M. (1989) L'Image, Paris, Editions VM.

Garrod S., Sanford A. J. (1988) Thematic Subjecthood and Cognitive Constraints on Discourse Structure, Journal of Pragmatics, 12, pp. 519-534.

Garvey C., Caramazza A. (1974) Implicit Causality in Verbs, Linguistic Inquiry, 5, pp. 459-464.

Grice H. P. (1975) Logic and Conversation, In Syntax and Semantics, Cole P., Morgan J. (eds.), Vol. 3, Academic Press, pp. 41-58.

Grosz B. J., Joshi A. K., Weinstein S. (1995) Centering: A Framework for Modelling the Local Coherence of Discourse, Computational Linguistics, 21:2, pp. 203-225.

Gundel J. K., Hedberg N., Zacharski R. (1993) Cognitive Status and the Form of Referring Expressions in Discourse, Language, 69:2, pp. 274-307. 
Halliday M. A. K. (1967) Notes on Transitivity and Theme in English, Part 2, Journal of Linguistics, 3:2, pp. 199-244.

Itten J. (1985) Art de la couleur, Paris, Dessain et Tolra.

Kessler K., Duwe I., Strohner H. (1996) Sprachliche Objektidentifikation in ambigen Situationen : Empirische Befunde, SFB 360 Situierte künstliche Kommunikation, Report 96/1, Universität Bielefeld.

Krahmer E., Theune M. (2002) Efficient Context-Sensitive Generation of Referring Expressions, In Information Sharing. Reference and Presupposition in Language Generation and Interpretation, Van Deemter K., Kibble R. (eds.), Stanford, CSLI Publications, pp. 223-264.

Lambrecht K. (1994) Information Structure and Sentence Form. Topic, Focus and the Mental Representations of Discourse Referents, Cambridge, Cambridge University Press.

Lambrecht K. (1996) On the Formal and Functional Relationship between Topics and Vocatives. Evidences from French, In Conceptual Structure, Discourse and Language, Goldberg A. E. (ed.), Stanford, CSLI Publications, pp. 267-288.

Landragin F. (2004) Dialogue homme-machine multimodal, Paris, Lavoisier.

Landragin F., Salmon-Alt S., Romary L. (2002) Ancrage référentiel en situation de dialogue, Traitement Automatique des Langues, 43, 2, pp. 99-129.

Lappin S., Leass H. J. (1994) A Syntactically Based Algorithm for Pronominal Anaphora Resolution, Computational Linguistics, 20:4, pp. 535-561.

Lyons J. (1970) Linguistique générale. Introduction à la linguistique théorique, Paris, Larousse.

Lyons J. (1980) Sémantique linguistique, Paris, Larousse.

McKoon G., Ward G., Ratcliff R., Sproat R. (1993) Morphosyntactic and Pragmatic Factors Affecting the Accessibility of Discourse Entities, Journal of Memory and Language, 32, pp. 56-75.

Miller G. A. (1956) The Magical Number Seven, Plus or Minor Two: Some Limits on our Capacity for Processing Information, Psychological Review, 63, pp. 81-97.

Osgood C. E., Bock J. K. (1977) Salience and Sentencing: Some Production Principles, In Rosenberg S. (Ed.) Sentence Production: Developments in Research and Theory, Hillsdale, Erlbaum, pp. 89-140. Pattabhiraman T. (1993) Aspects of Salience in Natural Language Generation, Ph.D. Thesis, Simon Fraser University.

Pattabhiraman T., Cercone N. (1990) Selection: Salience, Relevance and the Coupling between Domain-Level Tasks and Text Planning, In Proceedings of the Fifth International Workshop on Natural Language Generation, Dawson, pp. 79-86.

Pearson J., Poesio M., Stevenson R. (2001) The Effects of Animacy, Thematic Role and Surface Position on the Focusing of Entities in Discourse, In Proceedings of the First Workshop on Cognitively Plausible Models of Semantic Processing, Edinburgh.

Prince E. F. (1981) Toward a Taxonomy of Given-New Information, In Radical Pragmatics, Cole P. (ed.), New York, Academic, pp. 223-255.

Pylyshyn Z. W. (2001) Visual Indexes, Preconceptual Objects, and Situated Vision, Cognition, 80, $1 / 2$, pp. 127-158.

Rastier F. (1991) Sémantique et recherches cognitives, Paris, PUF.

Reboul A., Moeschler J. (1998) Pragmatique du discours. De l'interprétation de l'énoncé à l'interprétation du discours, Paris, Armand Colin. 
Reiter E., Dale R. (1997) Building Applied Natural-Language Generation Systems, Journal of Natural-Language Engineering, 3, pp. 57-87.

Rossi M. (1999) L'Intonation, le système du français. Description et modélisation, Paris, Ophrys.

Rousselet G. A., Fabre-Thorpe M. (2003) Les mécanismes de l'attention visuelle, Psychologie Française, 48, 1, pp. 29-44.

Sanmiguel D. (2000) Perspective et composition, Paris, Gründ.

Segall M. H., Campbell D. T., Herskovits M. J. (1966) The Influence of Culture on Visual Perception, Indianapolis, Bobbs-Merrill.

Sidner C. L. (1979) Towards a Computational Theory of Definite Anaphora in English Discourse, Ph.D. Thesis, MIT.

Sperber D., Wilson D. (1995) Relevance. Communication and Cognition (second edition), Oxford, Blackwell.

Stevenson R. J. (2002) The Role of Salience in the Production of Referring Expressions, In van Deemter K., Kibble R. (Eds.) Information Sharing: Reference and Presupposition in Language Generation and Interpretation, Stanford, CSLI Publications, pp. 167-192.

Stevenson R. J., Crawley R. A., Kleinman D. (1994) Thematic Roles, Focus and the Representation of Events, Language and Cognitive Processes, 9:4, pp. 519-548.

Van Dijk T. A. (1985) Structures of News in the Press, In Discourse and Communication, Van Dijk T. A. (ed.), Berlin, De Gruyter, pp. 69-93.

Van Kleeck M. H., Hillger L. A., Brown R. (1988) Pitting Verbal Schemas against Information Variables in Attribution, Social Cognition, 6, pp. 89-106.

Vettraino-Soulard M.-C. (1993) Lire une image, Paris, Armand Colin.

Victorri B., Fuchs C. (1996) La Polysémie. Construction dynamique du sens, Paris, Hermes.

Wanner L., Bateman J. A. (1990) A Collocational Based Approach to Salience-Sensitive Lexical Selection. In Proceedings of the Fifth International Workshop on Natural Language Generation, Dawson, pp. 31-38.

Wertheimer M. (1923) Untersuchungen zur Lehre von der Gestalt II, Psychologische Forschung, 4.

Wolters M. K. (2001) Towards Entity Status, Ph.D. Thesis, Bonn University.

\section{RÉSUMÉS}

Les phénomènes de saillance mettent en avant un élément d'un message linguistique ou visuel. En confrontant des travaux issus de disciplines variées, nous proposons une classification des facteurs qui déterminent la saillance d'une entité dans un énoncé linguistique ou dans une scène visuelle. Certains de ces facteurs ne dépendent que des caractéristiques physiques du message : on parlera alors de saillance physique (ou P-saillance). D'autres facteurs dépendent des processus cognitifs du sujet traitant le message : on parlera alors de saillance cognitive (ou C-saillance). A partir de cette classification, nous montrons que les notions de P-saillance et de C-saillance ne sont pas tributaires de la modalité (linguistique ou visuelle) à laquelle on les applique. Ceci nous permet d'aborder une caractérisation générique de la saillance physique et d'en tirer des conclusions sur la notion de structure informationnelle. 
Salience phenomena put an element from a linguistic or visual message forward. By confronting works resulting from various research fields, we propose a classification of the factors that make an entity salient in a linguistic utterance or in a visual scene. Some of these factors depend only on the physical characteristics of the message. We then talk about physical salience (or Psalience). Other factors depend on the subject's cognitive processes. We then talk about cognitive salience (or C-salience). Considering this classification, we show that P-salience and C-salience concepts do not rely on the modality (linguistic or visual) to which they apply. This allows us to lay the foundations for a generic characterization of physical salience, and to draw some conclusions concerning the information structure concept.

\section{INDEX}

Mots-clés : saillance, structure informationnelle, focus, thème, topique, perception visuelle

\section{AUTEUR}

\section{FRÉDÉRIC LANDRAGIN}

THALES Recherche et Technologie France, Domaine de Corbeville, 91404 Orsay Cedex 